\title{
A ventral salience network in the macaque brain
}

\author{
Alexandra Touroutoglou a,b,*,1, Eliza Bliss-Moreau ${ }^{\text {c,d,1 }}$, Jiahe Zhang ${ }^{\text {e,1 }}$, Dante Mantini f,g,h , Wim Vanduffel ${ }^{\text {b,f,i }}$, \\ Bradford C. Dickerson ${ }^{\mathrm{a}, \mathrm{b}}$, Lisa Feldman Barrett ${ }^{\mathrm{b}, \mathrm{e}, \mathrm{j}, * * *}$
}

a Department of Neurology, Massachusetts General Hospital/Harvard Medical School, 02114, USA

${ }^{\mathrm{b}}$ Athinoula A. Martinos Center for Biomedical Imaging, Massachusetts General Hospital/Harvard Medical School, MA 02129, USA

c California National Primate Research Center, Davis, CA 95616, USA

${ }^{\mathrm{d}}$ University of California at Davis, Davis, CA 95616, USA

e Northeastern University, Boston, MA 02115, USA

${ }^{\mathrm{f}}$ Laboratory for Neuro- and Psychophysiology, Department of Neurosciences, KU Leuven, 3000 Leuven, Belgium

${ }^{g}$ Department of Experimental Psychology, Oxford University, Oxford OX1 3UD, UK

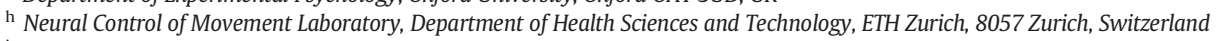

${ }^{i}$ Department of Radiology, Massachusetts General Hospital/Harvard Medical School, 02114, USA

${ }^{j}$ Department of Psychiatry, Massachusetts General Hospital/Harvard Medical School, 02129, USA

\section{A R T I C L E I N F O}

\section{Article history:}

Received 3 September 2015

Accepted 9 February 2016

Available online 17 February 2016

\section{Keywords:}

Intrinsic functional connectivity

Resting state fMRI

Salience network

Rhesus macaques

\begin{abstract}
A B S T R A C T
Successful navigation of the environment requires attending and responding efficiently to objects and conspecifics with the potential to benefit or harm (i.e., that have value). In humans, this function is subserved by a distributed large-scale neural network called the "salience network". We have recently demonstrated that there are two anatomically and functionally dissociable salience networks anchored in the dorsal and ventral portions of the human anterior insula (Touroutoglou et al., 2012). In this paper, we test the hypothesis that these two subnetworks exist in rhesus macaques (Macaca mulatta). We provide evidence that a homologous ventral salience network exists in macaques, but that the connectivity of the dorsal anterior insula in macaques is not sufficiently developed as a dorsal salience network. The evolutionary implications of these finding are considered.
\end{abstract}

(c) 2016 Elsevier Inc. All rights reserved.

\section{Introduction}

Salience processing is crucial for survival. It enables animals to successfully navigate the detection of, attention to, and action planning for stimuli that are potentially rewarding or harmful (i.e., that are relevant for allostasis). Allostasis, as the continually adjustment of the body's internal milieu to promote survival and reproduction, is a fundamental feature of the mammalian nervous system (Sterling, 2012; Sterling and Laughlin, 2015). Affect, characterized as valence (hedonicity) and arousal (physiological activation), is a cue to the value of stimuli for allostasis (Barrett and Bliss-Moreau, 2009) and is also thought to be a general feature of the mammalian nervous system (Anderson and Adolphs, 2014). The broadly distributed neural networks that subserve salience should, then, be present, in some form, in all mammals, but the existence of such networks across mammalian species remains an open question.

\footnotetext{
* Correspondence to: A. Touroutoglou, Department of Neurology, Harvard Medical School, Boston, MA 02114, USA.

** Correspondence to: L.F. Barrett, Department of Psychology, 125 Nightingale Hall, Northeastern University, Boston, MA 02115-5000, USA

${ }^{1}$ Authors made equal contributions.
}

A "salience network" (SN) has been identified within the intrinsic architecture of the human brain (Seeley et al., 2007) and its function linked to affect and attention (Barrett and Satpute, 2013; Touroutoglou et al., 2012). Major hubs of the salience network, including anterior insula (AI), anterior cingulate cortex (ACC), and amygdala, show spontaneous, low frequency blood oxygen level-dependent (BOLD) activity that fluctuates in a correlated manner in task independent periods (i.e., in the absence of external stimuli or tasks). We recently demonstrated (Touroutoglou et al., 2012) that the SN can be decomposed into two subnetworks that together represent salience in humans (see Fig. 1). Other neuroimaging studies have shown similar distinctive patterns of connectivity within the dorsal and ventral anterior insula (Chang et al., 2013; Deen et al., 2011; Kelly et al., 2012; Kurth et al., 2010; Taylor et al., 2009; Uddin et al., 2014). The ventral salience subnetwork, anchored in the agranular ventral $\mathrm{AI}(\mathrm{VAI})$, is connected to visceromotor regions that regulate allostasis, as well as regions that represent interoceptive and other sensory inputs linked to affective experience. Connectivity strength variation in this subnetwork uniquely predicted affective experience intensity when viewing unpleasant images (Touroutoglou et al., 2012). In contrast, the dysgranular dorsal anterior $\mathrm{AI}(\mathrm{dAI})$ anchors the dorsal salience network; this network is similar to the so-called ventral attention network (Corbetta et al., 2008; Corbetta and Shulman, 2002). Connectivity strength variation in 

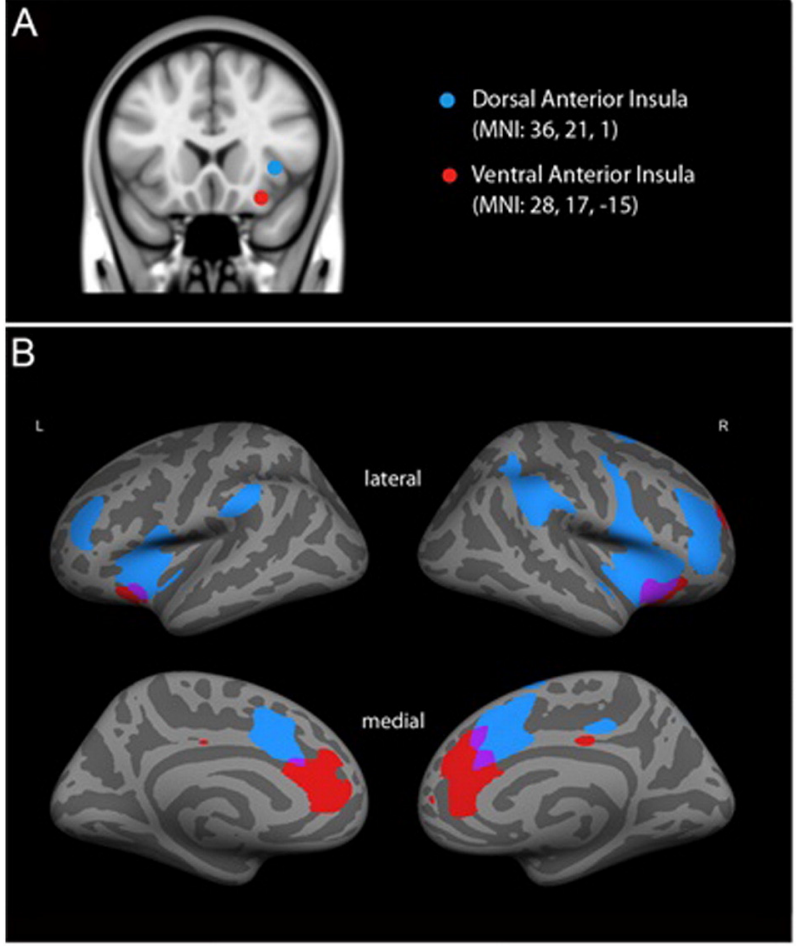

Dorsal Salience Network
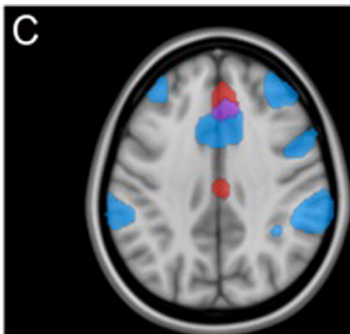

$z=36$

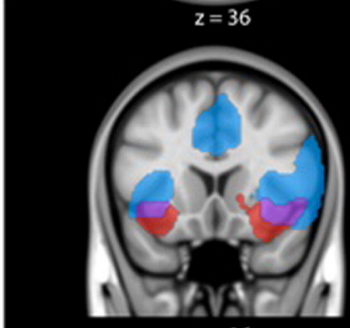

$y=16$

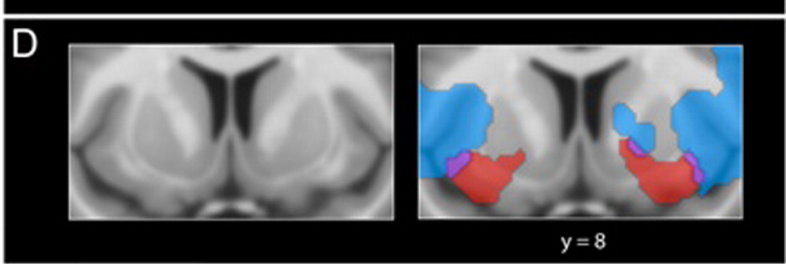

Ventral Salience Network

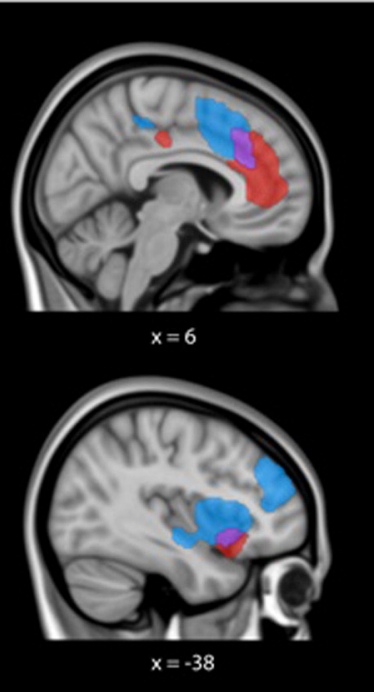

Overlap

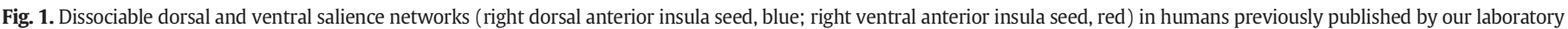

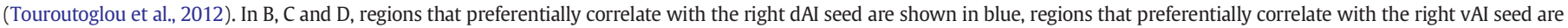

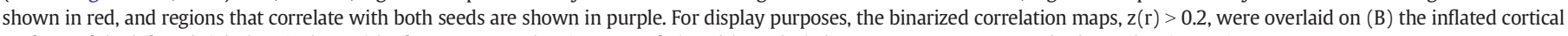
surfaces of the left and right hemispheres (the fsaverage template in FreeSurfer) and (C and D) the 1 mm MNI152 T1-standard template image in FSL.

Adapted figure from Touroutoglou et al., 2012.

this subnetwork predicted attentional processing-people with greater connectivity were better at switching their attention between sets (Touroutoglou et al., 2012). Thus, the SN can be thought of as an integrated system in which affect and attention interact to encode sensory stimuli that, in the past, have had allostatic consequences.

There has been extensive study of the neurobiological systems that support attentional processing in macaques (for reviews, see Desimone and Duncan, 1995; Squire et al., 2013). Also, a good deal is known about the macaque neural systems that code for value (i.e., whether a stimulus has been disruptive to allostasis in the past) (for reviews see Wallis, 2007; Morrison and Salzman, 2010). Yet, there is still much to learn about how value signals entrain attention to incoming sensory inputs that have been important to allostasis in the past (this has been called "salience"). Some regions that regulate allostasis (i.e., active during reward processing) are also active during spatial attention, such as ventromedial prefrontal cortex, ACC (Kaping et al., 2011) and the amygdala (Peck et al., 2013). Bidirectional anatomical connections (Mesulam and Mufson, 1982a; Mufson and Mesulam, 1982) and intrinsic connections (Hutchison et al., 2011, 2012) between two major nodes of the salience network, AI and ACC, have been identified in macaques, but studies have thus far failed to identify a fully developed salience network in monkeys (Mantini et al., 2013). Failure to find any evidence of comparable SNs would call into question the use of macaques as a good model for human brain function, as well as limit their translational value for studying human diseases in which salience or the anatomy or connectivity of the $\mathrm{SN}$ is perturbed (e.g., multiple neuropsychiatric disorders (Menon and Uddin, 2010; Uddin, 2014)).

In this paper, we tested the hypothesis that a homologous ventral salience subnetwork exists in macaques, but that a dorsal salience subnetwork would be less evident. We used a "seed-based" analysis, specifying two regions in anterior insula as anchor regions (Biswal et al., 1995; Vincent et al., 2007). We hypothesized that the networks originating at these seeds would reveal a homologous ventral network but not dorsal network given the evolutionary patterns of cortical expansion in humans relative to macaques (e.g., Hill et al., 2010; Preuss, 2012; Sherwood et al., 2012) and, in particular, the cortical layers in which the expansion is thought to be focused (Finlay and Uchiyama, 2014). That is, we reasoned that structures that constitute the ventral salience network are largely homologous across macaques and humans, while the dorsal salience network in humans included areas of frontal and parietal cortices that are substantially less developed in macaques (Orban et al., 2004; Passingham, 2009; Vanduffel et al., 2002).

\section{Materials and methods}

Subjects

Subjects were four rhesus macaques (Macaca mulatta, one female, 4-6 kg, 4-7 years old) who had been extensively trained and tested for other magnetic resonance imaging (MRI) studies (Mantini et al., 2012a, 2012b, 2011, 2013). Animal care standards were maintained according to all Belgian and European guidelines (European Union Directive on the Protection of Animals Used for Scientific Purposes 2010/63/EU). Experimental procedures were approved by the KU Leuven Medical School. Animals were socially housed (in pairs or small groups) and provided access to a large group socialization enclosure equipped with toys and enrichment devices. The monkeys received food ad libitum and were allowed to drink water until satiated during the experimental tests.

\section{Resting state procedure}

The current analyses used the same data as in Mantini et al. (2013). Briefly, monkeys were first trained to continuously fixate on a point (red 
dot centered on screen $0.3^{\circ}$ visual angle in size) on a blank screen in a mock scanner until they reached criterion of at least $95 \%$ fixation performance (Vanduffel et al., 2001). Once they reached criterion, they advanced to scanning sessions during which they performed the fixation task while MRI data was collected. During the fixation tasks, monkeys were rewarded with juice when they were successfully fixating. Immediately prior to fMRI data acquisition, animals were injected with microcrystalline iron-oxide nanoparticles (Sinerem; Guerbet; $6-10 \mathrm{mg} / \mathrm{kg}$ ) to increase signal-to-noise ratio beyond that of the normal BOLD signal. During each scan, monkeys sat in a sphinx position in a custom nonhuman primate chair that allowed their heads to be affixed to the chair via implanted MRI compatible head-posts (see Vanduffel et al., 2001). Each subject completed twenty 10-min resting state scans on 5 or 6 different occasions over a period of 6 months.

\section{fMRI data acquisition}

Data were collected on a 3 T Siemens Trio scanner in Leuven, Belgium (additional details can be found in Mantini et al., 2013). Monkeys were imaged using an in-house built 8-channel head coil with SENSE reconstruction and a saddle-shaped, radial transmitonly surface coil made specifically for use with nonhuman primates (Kolster et al., 2009). The functional images were collected using a gradient-echo T2*-weighted echo-planar sequence (40 slices, $84 \times 84$ in-plane matrix, TR/TE $=2000 / 19 \mathrm{~ms}$, flip angle $=75^{\circ}$, voxel size $=1.25 \times 1.25 \times 1.25 \mathrm{~mm}^{3}$ ). Additionally, T1-weighted anatomical scans were collected during different scanning sessions while animals were sedated with ketamine/xylazine (induction ketamine $10 \mathrm{mg} / \mathrm{kg}$ I.M. + Xylazine $0.5 \mathrm{mg} / \mathrm{kg}$ I.M., maintenance dose of 0.01 to $0.05 \mathrm{mg}$ ketamine per minute I.V.). Nine whole brain volumes were collected with a MP-RAGE sequence (TR/TE $=2200 / 4.06$, voxel size $=0.5 \times 0.5 \times 0.5 \mathrm{~mm}^{3}$ ) and averaged to create each animal's anatomical composite.

\section{fMRI data preprocessing}

Preprocessing of the resting state fMRI data involved a series of standard procedures previously described in Mantini et al. (2011). Preprocessing was performed with the SPM5.0 software package (http:// www.fil.ion.ucl.ac.uk/spm). These steps were: 1) slice-dependent time shifts correction, 2) motion correction, 3) linear detrending, 4) coregistration to the anatomical image, and 5) spatial normalization to macaque F99 (http://sumsdb.wustl.edu/sums/macaquemore.do) atlas space using the 112RM template (http://www.brainmap.wisc.edu/ monkey.html), and 6) spatial smoothing with a Gaussian kernel at $2 \mathrm{~mm}$ full-width-half-maximum. The method is the same as that used in Mantini et al. (2011) -in addition to signal detrending, cerebral spinal fluid and white matter signals and their first derivatives were regressed out of the signal. We did not elect to perform global signal regression. While there is a general consensus about the use of white matter and cerebrospinal fluid regression (Giove et al., 2009; Van Dijk et al., 2010), the importance of global signal regression (i.e. the average signal across the brain) is still debated. It has been demonstrated that the use of global signal regression potentially alters the wholebrain distribution of connectivity values, since the average correlation across the brain is forced to zero. This means that negative correlations may artifactually emerge as a result of global signal regression (Gotts et al., 2013; Murphy et al., 2009; Saad et al., 2012). Other studies disagree, and suggest that the global signal may be at least in part of artifactual origin (He \& Liu, 2012) so that the negative correlations that emerge after global signal regression are physiologically meaningful (Fox et al., 2009). Given the controversy, we decided to adopt a conservative approach and not perform global signal regression.

\section{Seed selection}

Salience network analyses were conducted by placing 2 seeds in the right anterior insula. Seed locations were selected based on evaluation of the macaque neuroanatomical literature (Mesulam and Mufson, 1982a, 1982b; Mufson and Mesulam, 1982) and two macaque brain atlases (Paxinos et al., 2000; Saleem and Logothetis, 2012) relative to a human brain atlas (Mai et al., 2008). Seed locations in the macaque brain were selected based on the established sulcal and gyral boundaries of the insula and other landmarks at the appropriate anterior-posterior levels such that they were at anatomically corresponding cortical locations as the human seed locations previously used to identify dorsal and ventral salience networks in two independent samples of healthy young adults $(\mathrm{N}=89$ and $\mathrm{N}=31$ ) (Touroutoglou et al., 2012). The dorsal and ventral seeds were spaced maximally so that they did not overlap, resulting in their placement slightly more dorsal and ventral than might have been expected based on Touroutoglou et al. (2012). Two spherical seeds (2-mm radius, as in Mantini et al., 2011) were used as regions of interest (ROIs), right vAI (17.5, 5.5, - 3.5) (Fig. 2) and right dAI $(20,2.5,1.5)$ (Fig. 3) (see Fig. 3S and Fig. 4 S in the Supplementary Materials for left vAI and left dAI seeds).

\section{Seed-based intrinsic connectivity network analyses}

\section{Spatial topography analysis}

We used whole brain seed-based analysis as used in Mantini et al. (2011) to explore the spatial topography of the intrinsic connectivity of VAI and dAI subregions. In a manner similar to seed-based intrinsic connectivity analyses previously published in nonhuman primates (Mantini et al., 2011; Vincent et al., 2007), we extracted the average signal across voxels within each seed region of interest (ROI) and calculated the correlations between the signals in the seed ROI and each voxel of the rest of the brain. Such methods stand in contrast to independent component analysis (Hutchison et al., 2012; Mantini et al., 2013; Moeller et al., 2009) which derives networks that are independent from each other by comparing correlated BOLD signal within and between networks using voxel-wise signals from the whole brain without regard to an anatomically defined point of origin (Joel et al., 2011). Individual whole brain connectivity maps were converted to $Z$-scores by the Fisher's $r$-to-z transformation. It is worth noting that the calculation of the $z$-scores in the present study differed from that in Touroutoglou et al. (2012) insofar as the calculations used here (from Mantini et al., 2011) account for the number of volumes per scan. As a result, the $\mathrm{z}$-scores from the present study are different than the z-scores used to threshold the previous human study (for equation of z-score calculation see also Supplementary Materials). Group correlation maps were created using a fixed-effect analysis (Genest, 1992). Similarly to the whole brain seed-based analysis used in Touroutoglou et al. (2012), we focused only on positive connectivity as both the dorsal and the ventral salience networks have previously shown positive correlations (Seeley et al., 2007; Touroutoglou et al., 2012).

The ventral and dorsal anterior insula maps were represented in the volume space and in the surface space. The conversion from volumes to surfaces was performed with Caret 5.61 (http://brainvis.wustl.edu/ wiki/index.php/Caret:Download) (Van Essen, 2012).

\section{Functional connectivity strength analysis}

To quantify the strength of intrinsic connectivity, we calculated the pair-wise correlations between each seed and its correlated target anatomical regions, as in Mantini et al. (2011). The target anatomical regions-those that showed connectivity with the vAI and dAI seeds-were determined through visual inspection of the dorsal salience and ventral salience maps independently, as in Touroutoglou et al. (2012), using the FreeSurfer viewing tool Freeview (http:// surfer.nmr.mgh.harvard.edu/). A minimum connectivity threshold was set to $z=2, p<0.0062$, corrected, with false discovery rate. After 

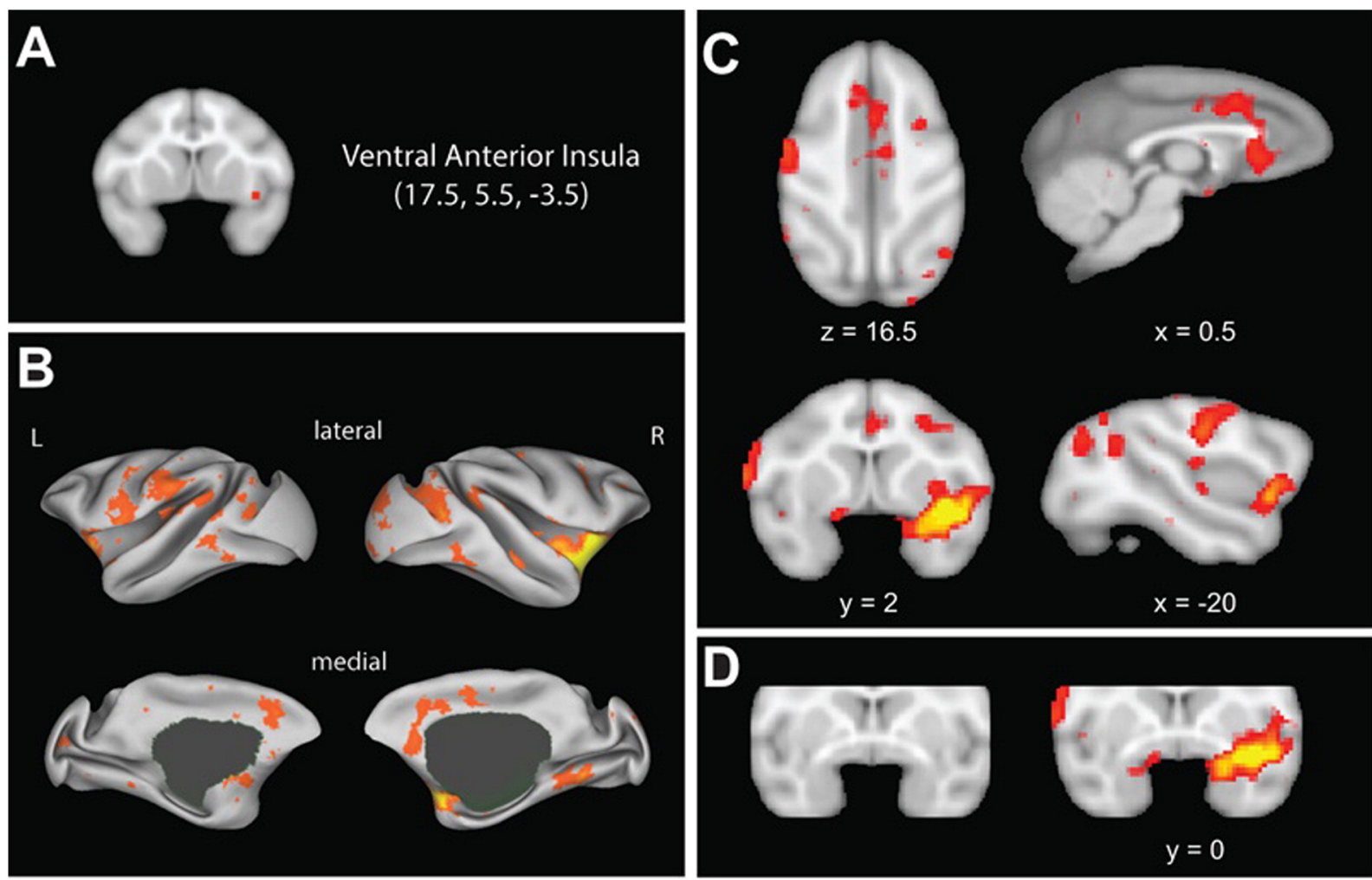

\section{0 $\quad 5.0$ Ventral Salience Network}

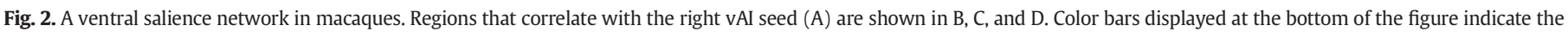

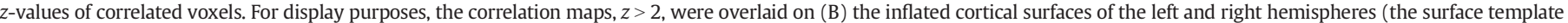
in Caret 5.61) and (C and D) the F99 T1 template in neurological convention.

localizing regions based on a combination of anatomically defined ROIs (Makris et al., 2010) and functional connectivity clusters, we quantified the connectivity strength for each ROI using a series of statistical steps previously published in Mantini et al. (2011).

To quantify the specificity of the dorsal and ventral salience networks, we compared within- and between-network connectivity. To do this, we computed two summary connectivity measures for each individual target region: 1) a dorsal anterior insula network connectivity measure, by averaging $z(\mathrm{r})$ values between the $\mathrm{dAI}_{\text {seed }}$ and each target region bilaterally across all monkeys; and 2) a ventral anterior insula network connectivity measure, by averaging $z(\mathrm{r})$ values between the $\mathrm{vAI}_{\text {seed }}$ and each target region bilaterally across all monkeys. Pairedsample t-tests compared the within- and between-network connectivity for both the dAI targets and vAI targets.

\section{Results}

\section{Connectivity for the ventral AI}

The topological correspondence of the ventral salience network between the monkey and the humans is presented in Fig. 1S in the Supplementary Materials. As in the human network (Touroutoglou et al., 2012), intrinsic BOLD signal within the macaque right vAI seed was correlated with intrinsic signal in the anterior and mid cingulate cortices [dorsal ACC (dACC), subgenual ACC (sgACC), mid cingulate cortex (MCC)], orbitofrontal cortex, amygdala, and putamen, as well as in the frontoinsula. As such, the network appeared to be largely homologous with the ventral salience network in humans. Unlike the human network, the macaque ventral salience network also included structures in temporal and dorsolateral striate cortex (e.g., superior temporal sulcus, lunate sulcus, etc.) as well as areas in frontal cortex (e.g., precentral and postcentral gyri) and mid and posterior insula (see Fig. 2). The mean and standard deviation of correlations between the vAI seed and the anatomical targets of the right ventral salience network across subjects are shown in Table 1S in the Supplementary Materials. Similar functional connectivity patterns were observed for the left vAI seed (see Fig. 3S in the Supplementary Materials).

\section{Connectivity for the dorsal AI}

The topological correspondence of the dorsal anterior insula network between the monkey and the humans is presented in Fig. $2 \mathrm{~S}$ in the Supplementary Materials. As in the human network, intrinsic BOLD signal within the macaque right $\mathrm{dAI}$ was correlated with intrinsic BOLD signal in other regions of the insula (bilateral vAI, mid and posterior insula), as well as areas in the frontal cortex located in the precentral and postcentral gyri, and areas of the cingulate. While the major anatomical nodes of the human network in the cingulate were the $\mathrm{dACC} / \mathrm{MCC}$, the macaque network dAI seed evidenced extensive connectivity with the bilateral sgACC and a cluster in posterior cingulate cortex (for functional connectivity of different subregions within the dAI see Fig. 7S in the Supplementary Materials; for functional connectivity of the mid-cingulate cortex see Fig. 8S in the Supplementary Materials). While both the human and macaque dAI seeds were connected to putamen, connectivity between the macaque dAI seed and putamen was much more extensive insofar as it was bilateral, the clusters in the putamen were large, and the connectivity strength between them and the dAI seed was high.

In contrast to the human network, which showed connectivity to the inferior parietal lobule, the supramarginal gyrus, and the middle frontal gyrus, the macaque dAI seed had connectivity only with the right intraparietal sulcus of the parietal cortex. In addition, the macaque dAI 

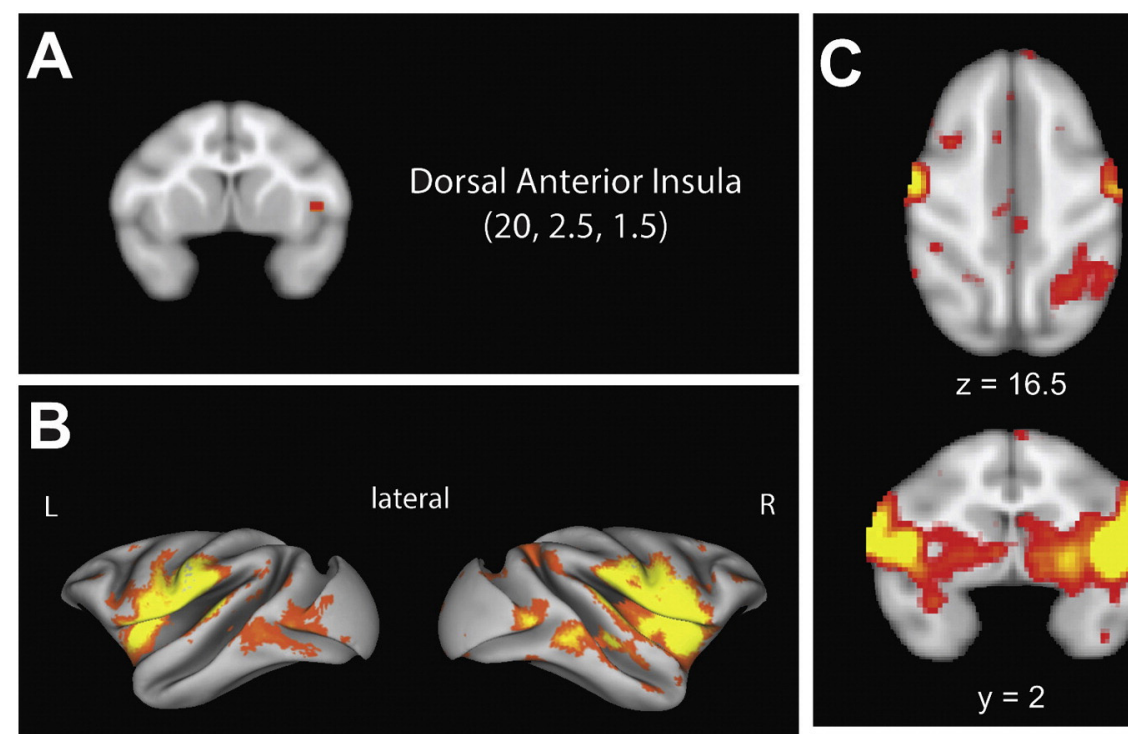

$z=16.5$

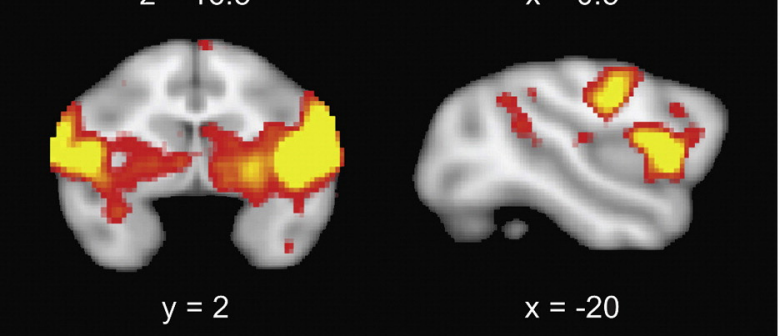

medial
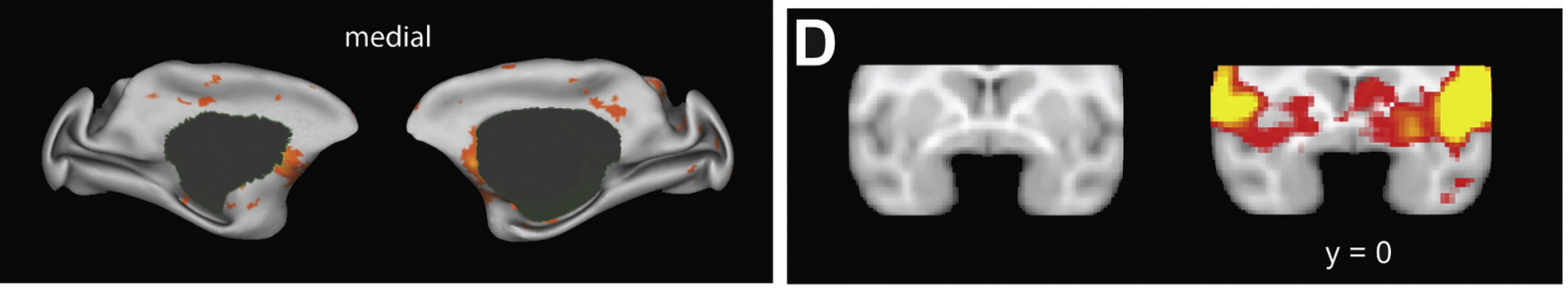

5.0

Dorsal Anterior Insula Network

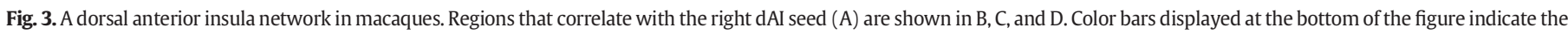

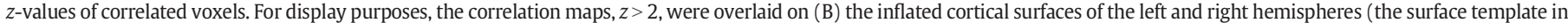
Caret 5.61) and (C and D) the F99 T1 template in neurological convention.

seed evidenced significant connections with areas of temporal and occipital cortices that were not present in the human network (Fig. 3). Another significant difference was the involvement of additional subcortical structures including the pallidum, basal forebrain, caudate, thalamus, hippocampus, and cerebellum (see Table $2 \mathrm{~S}$ in the Supplementary Materials). Similar functional connectivity patterns were observed for the left dAI seed (see Fig. $4 \mathrm{~S}$ in the Supplementary Materials).

A

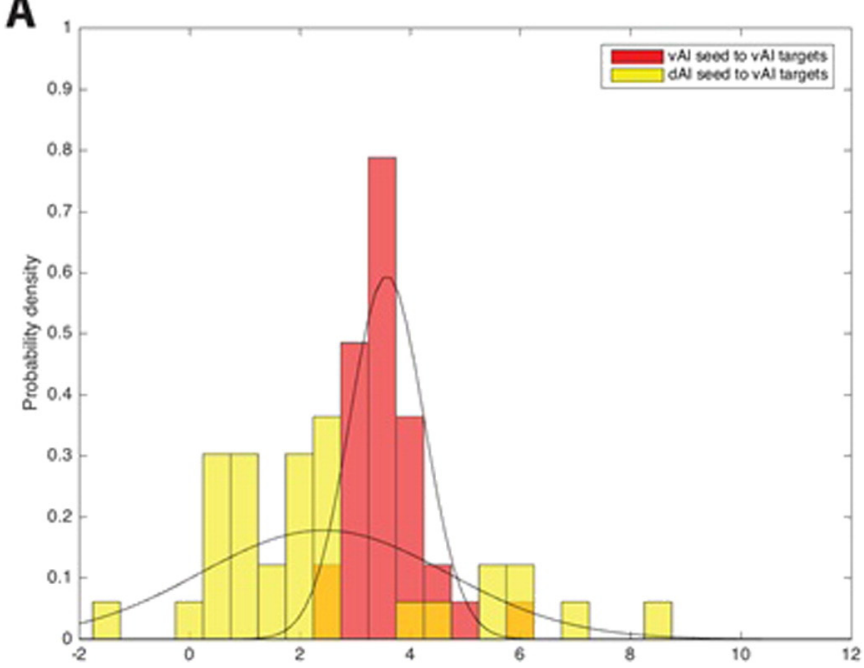

Dissociability of the ventral and dorsal AI connectivity

It was important to determine whether the networks that emerged in the macaque were dissociable from each other. Fig. 4 lists connectivity strength between the anterior insula seeds and their respective targets across four subjects [see also Fig. 2 in Touroutoglou et al. (2012) for comparison with the human data and the Supplementary Materials for a quality control analysis)].

B

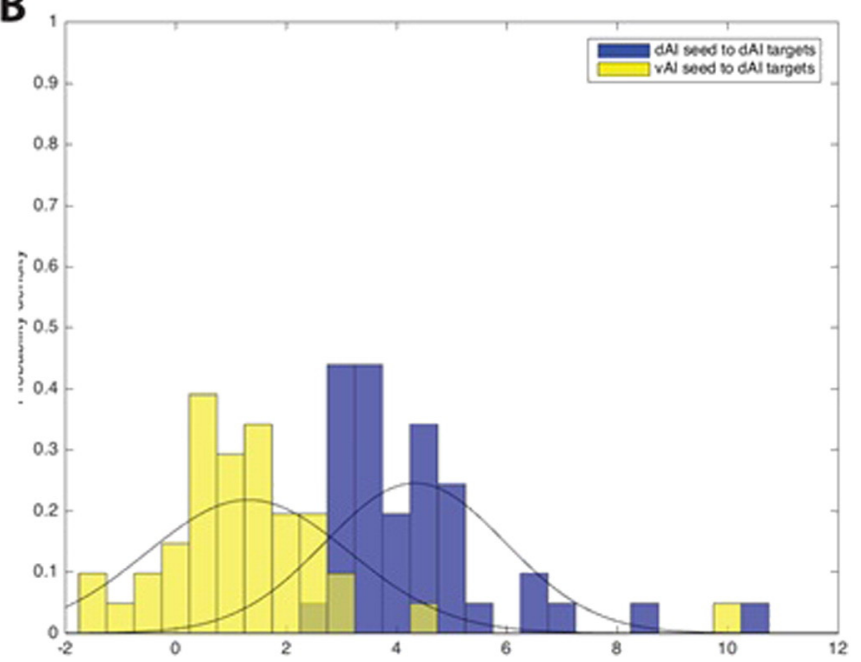

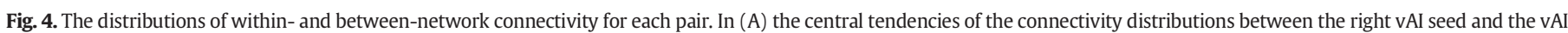

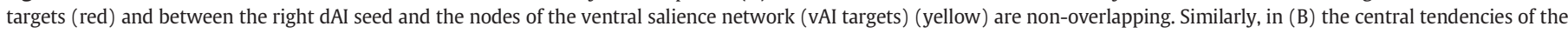


(yellow) are not overlapping. ROI pairs that are present in both the right dorsal and right ventral salience networks are indicated in dark yellow. 


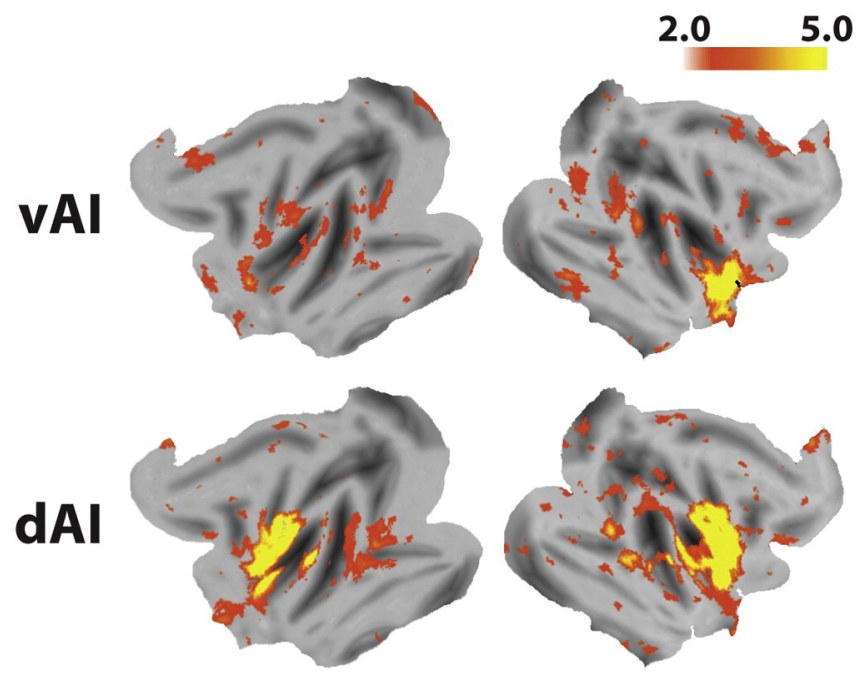

Fig. 5. The monkey ventral salience network and dorsal anterior insula network are represented over flattened cortical surfaces (threshold at $z>2$ ) (Caret 5.61).

The average within-network correlations were significantly stronger than the between network correlations providing evidence that networks are dissociable $\left[t(40)=9.952, p<0.001\right.$ for the $\mathrm{dAI}_{\text {seed }}-\mathrm{dAI}_{\text {targets }}$ as compared to $\mathrm{vAI}_{\text {seed }}-\mathrm{dAI}_{\text {targets }}$ connectivity and $t(32)=3.26$, $p<0.005$ for the $\mathrm{vAI}_{\text {seed }}-\mathrm{vAI}_{\text {targets }}$ as compared to $\mathrm{dAI}_{\text {seed }}-\mathrm{vAI}_{\text {targets }}$ connectivity].

While the human dorsal and ventral salience networks share some regions in common, the regions belonging to both subnetworks differed between species (see Fig. 5). In macaques, the ventral salience network and dAI connectivity both contained bilateral vAI, sgACC, and postcentral and precentral gyrus. In contrast, regions of overlap between the two salience subnetworks in the human included bilaterally the lateral OFC, vAI and adjacent frontal operculum, dACC/MCC and putamen (see Fig. 6; also see Fig. 5S for overlapping regions between the two salience networks at each individual monkey). A voxel wise statistical comparison of differences in the two subnetworks is presented in Fig. 6S in the Supplementary Materials.

\section{Discussion}

This study demonstrates that some, but not all, aspects of saliencerelated intrinsic connectivity are homologous across macaques and

ᄂ

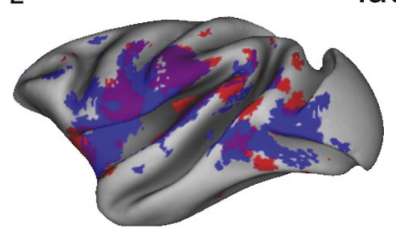

medial

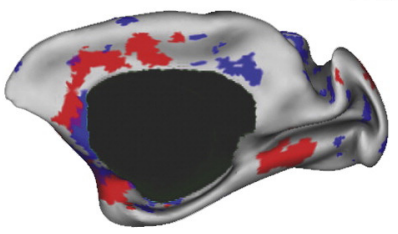

Dorsal Anterior Insula Network

Ventral Salience Network Overlap

Fig. 6. The monkey dorsal anterior insula network and ventral salience network are represented on the inflated cortical surfaces of the left and right hemispheres at $z=2$ (the surface template in Caret 5.61). Regions that preferentially correlate with the right $\mathrm{dAI}$ seed are shown in blue, regions that preferentially correlate with the right vAI seed are shown in red, and regions that correlate with both seeds are shown in purple.
Ventral Salience Network

human
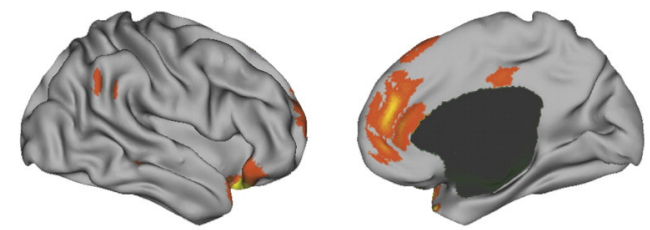

monkey
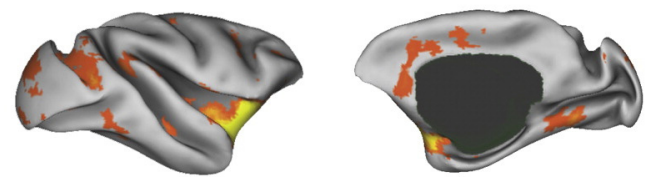

Fig. 7. A homologous ventral salience network in the human and macaque. Inflated cortical maps of the ventral salience network in humans $(z>.2)$ and monkeys $(z>2)$ plotted over a representative cortical surface in lateral and medial views.

humans. Using seed-based intrinsic connectivity analyses, we identified a homologous ventral salience subsystem generated by vAI that was anatomically dissociable from the connections to the dAI. The topography of the human and macaque ventral salience subnetworks was very similar (see Fig. 7).

The presence of a homologous ventral salience network is consistent with observations that representations of affect in macaques and humans share a number of striking similarities. For example, the parasympathetic and sympathetic branches of the macaque autonomic nervous system respond to evocative affective stimuli in a manner consistent with the responsivity of the human autonomic nervous system (Bliss-Moreau et al., 2013). Cardiac parasympathetic activity increased and sympathetic activity decreased as macaque subjects viewed videos stimuli that became less unpleasant and more pleasant. Similarly, individual differences in resting state (rather than taskevoked) cardiac activity in macaques relate to individual differences in affective processing (i.e., affective reactivity; Bliss-Moreau, in prep) as is the case in humans (e.g., (Licht et al., 2009, 2008; Oveis et al., 2009; Watkins et al., 1998). In humans, functional neuroimaging studies also indicate that activity within the VAI and other regions of salience network, such as amygdala and MCC, contributes to cardiac responsivity to evocative stimuli (Beissner et al., 2013; Craig, 2009; Critchley et al., 2004; Koelsch et al., 2007). We hypothesize that connectivity and activation in the ventral salience network are responsible for representing high-dimensional interoceptive changes (related to allostasis) as lower dimensional affective experience (Chanes and Barrett, 2016). Evidence for this hypothesis comes from studies documenting that stronger connectivity between vAI and pregenual ACC (Touroutoglou et al., 2012) as well as between the dorsal amygdala and the rest of the salience network (Touroutoglou et al., 2014) predict more intense selfreported arousal experience while viewing negative pictures. As such, it is possible that connectivity strength in the ventral salience network could be an index of affective experience in animals who cannot report on their experiences.

While there were some similarities in the connectivity associated with the macaque $\mathrm{dAI}$ seed and the human dorsal salience network, the lack of extensive connectivity with parietal and frontal regions suggests that the network that is associated with the $\mathrm{dAI}$ in the macaque is only a nascent homolog of the network associated with human dAI. These differences are likely the result of variation between macaque and human neuroanatomy. Macaque brains are thought to be comparable to human brains in terms of their general neuroanatomical regions, the structure of cortex, and global connections between brain areas (and arguably the best neuroscience model for human brains; Capitanio and Emborg, 2008; Phillips et al., 2014), but a number of substantial differences between macaque and human brains exist. It is clear, for example, that frontal and parietal areas associated with the dorsal salience network have grown in size and increased in connectivity 
in humans relative to macaques; the nature of those changes (e.g., whether or not the microstructure and connectivity of frontal and parietal regions have expanded in a way that is greater than what would be expected by scaling up of the brain alone (e.g., Finlay and Uchiyama, 2015; Herculano-Houzel, 2012; Hill et al., 2010; Orban et al., 2004; Passingham, 2009; Passingham and Smaers, 2014; Preuss, 2012; Rilling, 2006; Sherwood et al., 2012) is the source of considerable debate (see also Barton and Venditti, 2013; Vanduffel et al., 2014). It is possible that the expansion of insula connectivity with these parietal and other frontal regions in the human allows for the integration of salient information into some cognitive functions that appear to be unique to humans.

Our findings are consistent with growing evidence of intrinsic connectivity between the AI and the ACC in nonhuman primates (i.e., macaques: Hutchison et al., 2011, 2012; marmosets: Belcher et al., 2013) and therefore provide evidence that the choice of analysis method may be important in identifying cross-species similarities and differences. We found evidence of some salience network related connectivity in this macaque sample using a seed-based analysis method, whereas the original report utilizing ICA-based analytical methods on the same data (Mantini et al., 2013) did not. There are a number of methodological reasons why this might be the case, including sample size and requirements of filtering and univariate cross-correlations in seed-based approaches that are not requirements of ICA. The more important factor, however, was that our analyses were guided by prior findings about the meaningful dorsal-ventral dissociation in the salience network (Touroutoglou et al., 2014, 2012) combined with knowledge of anatomical differences between the human and macaque brains. Taken together, this combination led us to make specific apriori predictions about where we would find potential homologies across species. This seems particularly important when working with such small samples of subjects in nonhuman primate studies. Other methodological differences between prior studies in nonhuman primates, such as whether the animals are anesthetized (as in Hutchison et al., 2011, 2012), awake but resting without performing an attention task (as in Belcher et al., 2013), or awake and performing a fixation task (as in the present study and (Mantini et al., 2013)) are important to note, of course. These issues, however, now seem of secondary importance to the issue of whether or not a salience network can be found in the macaque brain.

Taken together, previous findings, in concert with our findings, point to clear homologies between the human and nonhuman primate salience subsystems that arise regardless of methodological variation. The findings are also consistent with new evidence that demonstrates that the microstructure of two major nodes in the SN, the vAI and dACC, may not differ between species as much as previously thought. It was long believed that macaques lacked "von Economo" neurons or "spindle cells" in the AI and ACC that are present in great apes (Allman et al., 2002). However, the presence of "von Economo" neurons in the AI has recently been documented in macaques (Evrard et al., 2012).

There are two logical next steps in this research program-one addressing a limitation of the present study and one expanding the scope of the investigation. First, the number of subjects in the present study is consistent with other nonhuman primate studies, but an important future direction is demonstrating the presence of the network in larger samples of macaques. In concert with demonstrating that the network is present in larger samples, a logical next step of this study is to investigate whether variation in intrinsic connectivity in the ventral salience network is associated with variation in affective processing in monkeys as it is in humans (Touroutoglou et al., 2012). To establish the link between ventral salience network connectivity and affect, it will be important to evaluate the same animals on tests of affective reactivity (e.g., induced changes to sympathetic and parasympathetic activity as in Bliss-Moreau et al., 2013) and network connectivity. After establishing that a relationship exists between affect and network connectivity, it would then be critical to evaluate the magnitude of the relationship between the two variables in order to understand how connectivity in the ventral salience network calibrates with affective experience. This provides an empirical approach for investigating affective processing in a wide-variety of animal species. The development of effective treatments and early interventions for affect-related psychopathology hinges on such animal models.

\section{Conclusions}

The salience network is important for encoding stimuli that have had allostatic relevance in the past (e.g., what is good, and what is bad). Using seed-based resting state functional connectivity analysis, we provide the first evidence that an affective salience network anchored in the ventral anterior insula exists in the macaque brain. The connectivity of the dorsal anterior insula in macaques did not constitute a network that is homologous to that observed in humans. Our results have important implications for translational systems neuroscience as they speak to issues of whether monkeys can experience affect and to the utility of non-human animal neuroimaging models of psychiatric illness.

\section{Acknowledgments}

We thank Dr. Ioannis Sanidas, Ph.D. for his assistance in preparing the figures of the manuscript and Olamide Abiose for her help in preparing the Supplementary Materials. Preparation of this manuscript was supported by funding from the National Institutes of Health: 5R01AG030311 to LFB and BCD and K99MH10138 to EBM; the Research Foundation Flanders (G0A5613, G083111), GOA/10/19; IUAP VII/11; PFV/10/008; the Hercules Foundation to WV; the Wellcome Trust and the Royal Society: Sir Henry Dale Fellowship grant $101253 / Z / 13 / Z$ to DM.

\section{Conflicts of interest}

The authors declare no competing financial interests.

\section{Appendix A. Supplementary data}

Supplementary data to this article can be found online at http://dx. doi.org/10.1016/j.neuroimage.2016.02.029.

\section{References}

Allman, J., Hakeem, A., Watson, K., 2002. Two phylogenetic specializations in the human brain. Neuroscientist 8, 335-346.

Anderson, David J., Adolphs, Ralph, 2014. A Framework for Studying Emotions across Species. Cell 157 (1), 187-200.

Barrett, L.F., Bliss-Moreau, E., 2009. Affect as a psychological primitive. Adv. Exp. Soc. Psychol. 41, 167-218.

Barrett, L.F., Satpute, A.B., 2013. Large-scale brain networks in affective and social neuroscience: towards an integrative functional architecture of the brain. Curr. Opin. Neurobiol. 1-12.

Barton, R.A., Venditti, C., 2013. Human frontal lobes are not relatively large. Proc. Natl. Acad. Sci. U. S. A. 110, 9001-9006.

Beissner, F., Meissner, K., Bar, K.J., Napadow, V., 2013. The autonomic brain: an activation likelihood estimation meta-analysis for central processing of autonomic function. J. Neurosci. 33, 10503-10511.

Belcher, A.M. Yen, C.C., Stepp, H., Gu, H., Lu, H., Yang Y., Silva, A.C., Stein, E.A., 2013. Largescale brain networks in the awake, truly resting marmoset monkey. J. Neurosci. 33 16796-16804.

Biswal, B., Yetkin, F.Z., Haughton, V.M., Hyde, J.S., 1995. Functional connectivity in the motor cortex of resting human brain using echo-planar MRI. Magn. Reson. Med Off. J. Soc. Mag. Reson. Med./Soc. Magn. Reson. Med. 34, 537-541.

Bliss-Moreau, E., Machado, C.J., Amaral, D.G., 2013. Macaque cardiac physiology is sensitive to the valence of passively viewed sensory stimuli. PLoS ONE 8, e71170.

Capitanio, J.P., Emborg, M.E., 2008. Contributions of non-human primates to neuroscience research. Lancet 371, 1126-1135.

Chanes, L., Barrett, L.F., 2016. Redefining the role of limbic areas in cortical processing. Trends Cogn. Sci. 20, 96-106. 
Chang, L.J., Yarkoni, T., Khaw, M.W., Sanfey, A.G., 2013. Decoding the role of the insula in human cognition: functional parcellation and large-scale reverse inference. Cereb. Cortex 23, 739-749.

Corbetta, M., Shulman, G.L., 2002. Control of goal-directed and stimulus-driven attention in the brain. Nat. Rev. Neurosci. 3, 201-215.

Corbetta, M., Patel, G., Shulman, G.L., 2008. The reorienting system of the human brain: from environment to theo... - PubMed - NCBI. Neuron 58, 306-324.

Craig, A.D., 2009. How do you feel-now? The anterior insula and human awareness. Nat Rev. Neurosci. 10, 59-70.

Critchley, H.D., Wiens, S., Rotshtein, P., Ohman, A., Dolan, R.J., 2004. Neural systems supporting interoceptive awareness. Nat. Neurosci. 7, 189-195.

Deen, B., Pitskel, N.B., Pelphrey, K.A., 2011. Three systems of insular functional connectivity identified with cluster analysis. Cereb. Cortex 21, 1498-1506.

Desimone, R., Duncan, J., 1995. Neural mechanisms of selective visual attention. Annu. Rev. Neurosci. 193-222.

Evrard, H.C., Forro, T., Logothetis, N.K., 2012. Von Economo neurons in the anterior insul of the macaque monkey. Neuron 74, 482-489.

Finlay, B.L., Uchiyama, R., 2014. Developmental mechanisms channeling cortical evolution. Trends Neurosci.

Finlay, B.L., Uchiyama, R., 2015. Developmental mechanisms channeling cortical evolution. Trends Neurosci. 38, 69-76.

Fox, M.D., Zhang, D., Snyder, A.Z., Raichle, M.E., 2009. The global signal and observed anticorrelated resting state brain networks. J. Neurophysiol. 101, 3270-3283.

Genest, C., 1992. Vincentization revisited. Ann. Stat. 20, 1137-1142.

Giove, F., Gili, T., Iacovella, V., VMacaluso, E., Maraviglia, B., 2009. Images-based suppression of unwanted global signals in resting-state functional connectivity studies. Magn. Reson. Imaging 27, 1058-1064.

Gotts, S.J., Saad, Z.S., Jo, H.J., Wallace, G.L., Cox, R.W., Martin, A., 2013. The perils of globa signal regression for group comparisons: a case study of autism spectrum disorders. Front. Hum. Neurosci. 7, 356

He, H., Liu, T.T., 2012. A geometric view of global signal confounds in resting-state functional MRI. Neuroimage 5, 2339-2348.

Herculano-Houzel, S., 2012. The remarkable, yet not extraordinary, human brain as a scaled-up primate brain and its associated cost. Proc. Natl. Acad. Sci. U. S. A. 109 (Suppl. 1), 10661-10668.

Hill, J., Inder, T., Neil, J., Dierker, D., Harwell, J., Van Essen, D., 2010. Similar patterns of cortical expansion during human development and evolution. Proc. Natl. Acad. Sci. U. S. A. 107, 13135-13140.

Hutchison, R.M., Leung, L.S., Mirsattari, S.M., Gati, J.S., Menon, R.S., Everling, S., 2011. Resting-state networks in the macaque at $7 \mathrm{~T}$. Neurolmage 56, 1546-1555.

Hutchison, R.M., Womelsdorf, T., Gati, J.S., Leung, L.S., Menon, R.S., Everling, S., 2012 Resting-state connectivity identifies distinct functional networks in macaque cingulate cortex. Cereb. Cortex 22, 1294-1308.

Joel, S.E., Caffo, B.S., van Zijl, P.C.M., Pekar, J.J., 2011. On the relationship between seedbased and ICA-based measures of functional connectivity. Magn. Reson. Med. 66, 644-657.

Kaping, D., Vinck, M., Hutchison, R.M., Everling, S., Womelsdorf, T., 2011. Specific contributions of ventromedial, anterior cingulate, and lateral prefrontal cortex for attentional selection and stimulus valuation. PLoS Biol. 9, e1001224.

Kelly, C., Toro, R., Di Martino, A., Cox, C.L., Bellec, P., Castellanos, F.X., Milham, M.P., 2012. convergent functional architecture of the insula emerges across imaging modalities. Neurolmage 61, 1129-1142.

Koelsch, S., Remppis, A., Sammler, D., Jentschke, S., Mietchen, D., Fritz, T., Bonnemeier, H. Siebel, W.A., 2007. A cardiac signature of emotionality. Eur. J. Neurol. 26, 3328-3338.

Kolster, H., Mandeville, J.B., Arsenault, J.T., Ekstrom, L.B., Wald, L.L., Vanduffel, W., 2009 Visual field map clusters in macaque extrastriate visual cortex. J. Neurosci. 29, 7031-7039.

Kurth, F., Zilles, K., Fox, P.T., Laird, A.R., Eickhoff, S.B., 2010. A link between the systems: functional differentiation and integration within the human insula revealed by meta-analysis. Brain Struct. Funct. 214, 519-534.

Licht, C.M.M., de Geus, E.J.C., Zitman, F.G., Hoogendijk, W.J.G., van Dyck, R., Penninx, B.W.J.H., 2008. Association between major depressive disorder and heart rate variability in the Netherlands study of depression and anxiety (NESDA). Arch. Gen. Psychiatry 65,1358 .

Licht, C.M.M., De Geus, E.J.C., van Dyck, R., Penninx, B.W.J.H., 2009. Association between anxiety disorders and heart rate variability in the Netherlands study of depression and anxiety (NESDA). Psychosom. Med. 71, 508-518.

Mai, J., Paxinos, G., Voss, T., 2008. Atlas of the Human Brain. third ed. Academic Press, San Diego, California.

Makris, N., Kennedy, D.N., Boriel, D.L., Rosene, D.L., 2010. Methods of MRI-based structural imaging in the aging monkey. Methods 50, 166-177.

Mantini, D., Gerits, A., Nelissen, K., Durand, J.-B., Joly, O., Simone, L., Sawamura, H., Wardak, C., Orban, G.A., Buckner, R.L., Vanduffel, W., 2011. Default mode of brain function in monkeys. J. Neurosci. 31, 12954-12962.

Mantini, D., Corbetta, M., Romani, G.L., Orban, G.A., Vanduffel, W., 2012a. Data-driven analysis of analogous brain networks in monkeys and humans during natural vision. Neurolmage 63, 1107-1118.

Mantini, D., Hasson, U., Betti, V., Perrucci, M.G., Romani, G.L. Corbetta, M., Orban, G.A. Vanduffel, W., 2012b. Interspecies activity correlations reveal functional correspondence between monkey and human brain areas. Nat. Methods 9, 277-282.

Mantini, D., Corbetta, M., Romani, G.L., Orban, G.A., Vanduffel, W., 2013. Evolutionarily novel functional networks in the human brain? J. Neurosci. 33, 3259-3275.

Menon, V., Uddin, L.Q., 2010. Saliency, switching, attention and control: a network mode of insula function. Brain Struct. Funct. 214, 655-667.
Mesulam, M.M., Mufson, EJ., 1982a. Insula of the old world monkey. I. Architectonics in the insulo-orbito-temporal component of the paralimbic brain. J. Comp. Neurol. $212,1-22$

Mesulam, M.M., Mufson, E.J., 1982b. Insula of the old world monkey. III: Efferent cortical output and comments on function. J. Comp. Neurol. 212, 38-52.

Moeller, S., Nallasamy, N., Tsao, D.Y., Freiwald, W.A., 2009. Functional connectivity of the macaque brain across stimulus and arousal states. J. Neurosci. 29, 5897-5909.

Morrison, S.E., Salzman, C.D., 2010. Re-valuing the amygdala. Curr. Opin. Neurobiol. 20, 221-230.

Mufson, E.J., Mesulam, M.M., 1982. Insula of the old world monkey. II: Afferent cortical input and comments on the claustrum. J. Comp. Neurol. 212, 23-37.

Murphy, K., Birn, R.M., Handwerker, D.A., Jones, T.B., Bandettini, P.A., 2009. The impact of global signal regression on resting state correlations: are anti-correlated networks introduced? NeuroImage 44, 893-905.

Orban, G.A., Van Essen, D., Vanduffel, W., 2004. Comparative mapping of higher visual areas in monkeys and humans. Trends Cogn. Sci. 8, 315-324.

Oveis, C., Cohen, A.B., Gruber, J., Shiota, M.N., Haidt, J., Keltner, D., 2009. Resting respiratory sinus arrhythmia is associated with tonic positive emotionality. Emotion 9, $265-270$

Passingham, R., 2009. How good is the macaque monkey model of the human brain? PubMed - NCBI. Curr. Opin. Neurobiol. 19, 6-11.

Passingham, R.E., Smaers, J.B., 2014. Is the prefrontal cortex especially enlarged in the human brain allometric relations and remapping factors. Brain Behav. Evol. 84, $156-166$.

Paxinos, G., Huang, X.-F., Toga, A.W., 2000. The Rhesus Monkey Brain in Stereotaxic Coordinates. Academic Press.

Peck, C.J., Lau, B., Salzman, C.D., 2013. The primate amygdala combines information about space and value. Nat. Neurosci. 16, 340-348.

Phillips, K.A., Bales, K.L., Capitanio, J.P., Conley, A., Czoty, P.W., t Hart, B.A., Hopkins, W.D. Hu, S.L., Miller, L.A., Nader, M.A., Nathanielsz, P.W., Rogers, J., Shively, C.A., Voytko, M.L., 2014. Why primate models matter. Am. J. Primatol. 76, 801-827.

Preuss, T.M., 2012. Human brain evolution: from gene discovery to phenotype discovery. Proc. Natl. Acad. Sci. U. S. A. 109 (Suppl. 1), 10709-10716.

Rilling, J.K., 2006. Human and nonhuman primate brains: are they allometrically scaled versions of the same design? Evol. Anthropol. Issues Rev. Rev. 15, 65-77.

Saad, Z.S., Gotts, S.J., Murphy, K., Chen, G., Jo, H.J., Martin, A., Cox, R.W., 2012. Trouble at rest: how correlation patterns and group differences become distorted after global signal regression. Brain Connect 2, 25-32.

Saleem, K.S., Logothetis, N.K., 2012. A Combined MRI and Histology Atlas of the Rhesus Monkey Brain in Stereotaxic Coordinates. Elsevier.

Seeley, W.W., Menon, V., Schatzberg, A.F., Keller, J., Glover, G.H., Kenna, H., Reiss, A.L., Greicius, M.D., 2007. Dissociable intrinsic connectivity networks for salience processing and executive control. J. Neurosci. 27, 2349-2356.

Sherwood, C.C., Bauernfeind, A.L., Bianchi, S., Raghanti, M.A., Hof, P.R., 2012. Human brain evolution writ large and small. Prog. Brain Res. 195, 237-254.

Squire, R.F., Noudoost, B., Schafer, R.J., Moore, T., 2013. Prefrontal contributions to visual selective attention. Annu. Rev, Neurosci. 36, 451-466.

Sterling, P., 2012. Allostasis: a model of predictive regulation. Physiol. Behav. 106, 5-15

Sterling, P., Laughlin, S., 2015. Principles of Neural Design. The MIT Press, Cambridge, Massachusetts.

Taylor, K.S., Seminowicz, D.A., Davis, K.D., 2009. Two systems of resting state connectivity between the insula and cingulate cortex. Hum. Brain Mapp. 30, 2731-2745.

Touroutoglou, A., Hollenbeck, M., Dickerson, B.C., Barrett, L.F., 2012. Dissociable largescale networks anchored in the right anterior insula subserve affective experience and attention. Neurolmage 60, 1947-1958.

Touroutoglou, A., Bickart, K.C., Barrett, L.F., Dickerson, B.C., 2014. Amygdala task-evoked activity and task-free connectivity independently contribute to feelings of arousal. Hum. Brain Mapp. 35, 5316-5327.

Uddin, L.Q., 2014. Salience processing and insular cortical function and dysfunction. Nat. Rev. Neurosci.

Uddin, L.Q., Kinnison, J., Pessoa, L., Anderson, M.L., 2014. Beyond the tripartite cognitionemotion-interoception model of the human insular cortex. J. Cogn. Neurosci. 26, $16-27$.

Van Dijk, K.R, Hedden, T, Venkataraman, A Evans, K.C. Lazar, S.W Buckner, R.L, 2010. Intrinsic functional connectivity as a tool for human connectomics: theory, properties, and optimization. J. Neurophysiol. 103, 297-321

Van Essen, D.C., 2012. Cortical cartography and Caret software. Neurolmage 62, 757-764. Vanduffel, W., Fize, D., Mandeville, J.B., Nelissen, K., Van Hecke, P., Rosen, B.R., Tootell, R.B.H., Orban, G.A., 2001. Visual motion processing investigated using contrast agent-enhanced fMRI in awake behaving monkeys. Neuron 32, 565-577.

Vanduffel, W., Fize, D., Peuskens, H., Denys, K., Sunaert, S., Todd, J.T., Orban, G.A., 2002. Extracting 3D from motion: differences in human and monkey intraparietal cortex. Science 298, 413-415

Vanduffel, W., Zhu, Q., Orban, G.A., 2014. Monkey cortex through fMRI glasses. Neuron 83, 533-550.

Vincent, J.L., Patel, G.H., Fox, M.D., Snyder, A.Z., Baker, J.T., Van Essen, D.C., Zempel, J.M., Snyder, L.H., Corbetta, M., Raichle, M.E., 2007. Intrinsic functional architecture in the anaesthetized monkey brain. Nature 447, 83-86.

Wallis, J.D., 2007. Orbitofrontal cortex and its contribution to decision-making. Annu. Rev. Neurosci. 30, 31-56.

Watkins, L.L., Grossman, P., Krishnan, R., Sherwood, A., 1998. Anxiety and vagal control of heart rate. Psychosom. Med. 60, 498-502. 